\title{
ANOMALOUS U(1) GAUGE SYMMETRIES AND HETEROTIC - TYPE I/II STRING DUALITY
}

\author{
Hans Peter Nilles \\ Physikalisches Institut, Universität Bonn, Nussallee 12, D-53115 Bonn, Germany \\ E-mail: 'nilles@th.physik.uni-bonn.dé
}

ABstraCt: Anomalous U(1) gauge symmetries can appear both in heterotic and type I string theories. In the heterotic case we find a single anomalous $\mathrm{U}(1)$, while in open string theories several such symmetries can appear. Nonetheless, there is a conjectured duality symmetry that might connect these two theories. We review the properties of anomalous gauge symmetries in various string theories as well as the status of this heterotic-type I/II duality. We also comment on the possible phenomenological applications of anomalous gauge symmetries in string theory.

\section{Motivation}

In quantum field theory there are strong arguments against the consistency of anomalous gauge symmetries. However, they did not seem to carry over to the framework of string theory, at least in the $U(1)$ case. In fact, anomalous $\mathrm{U}(1)$ gauge symmetries appeared in many consistent string theories and have received considerable attention. Primarily the motivation to study such symmetries was of theoretical origin, trying to understand the explicit mechanism that made these theories acceptable. It was soon realized that there could be interesting applications to phenomenology as well. This included the possible role of induced Fayet-Iliopoulos terms for gauge and supersymmetry breakdown as well as the appearance of global symmetries relevant for the strong CP-problem and questions of baryon and lepton number conservation. Cosmological applications can be found in a discussion of Dterm inflation and the creation of the cosmological baryon asymmetry.

In the more theoretical studies, it was realized that anomalous $\mathrm{U}(1)$ gauge symmetries can serve as tools to study detailed properties of duality symmetries. Most recently this became ap- parent in attempts to relate orbifold compactifications of the perturbative heterotic string to orientifolds of Type II string theory. Here I shall report on results obtained in collaboration with Z. Lalak and S. Lavignac. Lack of space and time allows just a summary of basic results. For details and a more complete list of references we refer the reader to the original publications [i]1,

\section{Anomalous $U(1)$ gauge symmetry in heterotic string theory}

In field theoretic models we were taught to discard anomalous gauge symmetries in order to avoid inconsistencies. This was even extended for the condition on the trace of the charges $\sum_{i} Q_{i}=$ 0 of a $U(1)$ gauge symmetry because of mixed gauge and gravitational anomalies [3]. Moreover a nonvanishing trace of the $U(1)$ charges would reintroduce quadratic divergencies in supersymmetric theories through a one-loop FayetIliopoulos term [i] i In string theory we then learned that one can tolerate anomalous $U(1)$ gauge symmetries as a consequence of the appearance of the Green-Schwarz mechanism that provides a mass for the anomalous gauge boson. In fact, anomalous $U(1)$ gauge symmetries are common in string theories and could be useful for various reasons. In the case of the het- 
erotic string one obtains models with at most one anomalous $U(1)$, and the Green-Schwarz mechanism involves the so-called model independent axion (the pseudoscalar of the dilaton superfield $S)$. The number of potentially anomalous gauge bosons is in general limited by the number of antisymmetric tensor fields in the ten-dimensional $(d=10)$ string theory. This explains the appearance of only one such gauge boson in the perturbative heterotic string theory and leads to specific correlations between the various (mixed) anomalies [6]. This universal anomaly structure is tied to the coupling of the dilaton multiplet to the various gauge bosons. The appearance of a nonvanishing trace of the $U(1)$ charges leads to the generation of a Fayet-Ilopoulos term $\xi^{2}$ at one loop. In the low energy effective field theory this would be quadratically divergent, but in string theory this divergence is cut off through the inherent regularization due to modular invariance. One obtains [י]

$$
\xi^{2} \sim \frac{1}{\left(S+S^{*}\right)} M_{\text {Planck }}^{2} \sim M_{\text {String }}^{2}
$$

where $\left(S+S^{*}\right) \sim 1 / g^{2}$ with the string coupling constant $g$. The Fayet-Iliopoulos term of order of the string scale $M_{\text {String }}$ is thus generated in perturbation theory. This could in principle lead to a breakdown of supersymmetry, but in all known cases there exists a supersymmetric minimum in which charged scalar fields receive nonvanishing vacuum expectation values (vevs), that break the anomalous $\mathrm{U}(1)$ (and even other gauge groups) spontaneously. This then leads to a mixing of the goldstone boson (as a member of a matter supermultiplet) of this spontaneous breakdown and the model-independent axion (as a member of the dilaton multiplet) of the Green-Schwarz mechanism. One of the linear combinations will provide a mass to the anomalous gauge boson. The other combination will obtain a mass via nonperturbative effects that might even be related to an axion-solution of the strong CP-problem [9i]. As we can see from (12.1), both the mass of the $U(1)_{A}$ gauge boson and the value of the Fayet-Iliopoulos term $\xi$ are of the order of the string scale. Nonetheless, models with an anomalous $U(1)$ have been considered under various circumstances and lead to a number of desirable consequences. Among those are the breakdown of some additional nonanomalous gauge groups [101], a mechanism to parametrize the fermion mass spectrum in an economical way iij, the possibility to induce a breakdown of supersymmetry [12]', a satisfactory incorporation of D-term inflation [13], and the possibility for an axion solution of the strong CP-problem [i] property of the perturbative heterotic string theory in the presence of an anomalous $U(1)$ is the fact that both $\xi$ and the mass of the anomalous gauge boson are induced dynamically and not just put in by hand. Both of them, though, are of order of the string scale $M_{\text {String, }}$, which might be too high for some of the applications. We will now compare this for the case of type I and type II orientifolds.

\section{Anomalous $U(1)$ 's in type I and type II theories}

We consider $d=4$ string models of both open and closed strings that are derived from either type I or type II string theories in $d=10$ by appropriate orbifold or orientifold projections $[\overline{1} \overline{1} \mid$. It was noticed, that in these cases more than a single anomalous $U(1)$ symmetry could be obtained $\left[1{ }^{1}{ }_{1}\right]$. This lead to the belief that here we can deal with a new playground of various sizes of $\xi$ 's and gauge boson masses in the phenomenological applications.

The appearance of several anomalous $U(1)$ 's is a consequence of the fact that these models contain various antisymmetric tensor fields in the higher dimensional theory and the presence of a generalized Green-Schwarz mechanism [1] $\left.\overline{7}_{1}, 1 \overline{1}^{\prime}\right]$ involving axion fields in new supermultiplets $M$. In the type II orientifolds under consideration these new axion fields correspond to twisted fields in the Ramond-Ramond sector of the theory.

From experience with the heterotic case it was then assumed $\left[19_{1}^{\prime}\right]$ that for each anomalous $U(1)$ a Fayet-Iliopoulos term was induced dynamically. With a mixing of the superfields $M$ and the dilaton superfield $S$ one hoped for $U(1)_{A}$ gauge boson masses of various sizes in connection with various sizes of the $\xi$ 's.

The picture of duality between heterotic orbifolds and type II orientifolds as postulated in 
$[20-1]$ seemed to work even in the presence of several anomalous $U(1)$ gauge bosons assuming the presence of Fayet-Iliopoulos terms in perturbation theory and the presence of the generalized Green-Schwarz mechanism. So superficially everything seemed to be understood. But apparently the situation turned out to be more interesting than anticipated.

There appeared two decisive results that initiated renewed interest in these questions and forced us to reanalyse this situation [1]. The first one concerns the inspection of the anomaly cancellation mechanism in various type II orientifolds. As was observed by Ibáñez, Rabadan and Uranga [2 $\left.{ }^{2} \overline{1}\right]$, in this class of models there is no mixing between the dilaton multiplet and the $M$ fields. It is solely the latter that contribute to the anomaly cancellation. Thus the dilaton that is at the origin of the Green-Schwarz mechanism in the heterotic theory does not participate in that mechanism in the dual orientifold picture. The second new result concerns the appearance of the Fayet-Iliopoulos terms in type I theory. As was shown by Poppitz [202"] in a specific model, there were no $\xi$ 's generated in one-loop perturbation theory. The one loop contribution vanishes because of tadpole cancellation in the given theory. This result seems to be of more general validity and could have been anticipated from general arguments, since in type I theory a (one-loop) contribution to a Fayet-Iliopoulos term either vanishes or is quadratically divergent, and the latter divergence is avoided by the requirement of tadpole cancellation. Of course, there is a possibility to have tree level contributions to the $\xi$ 's, but they are undetermined, in contrast to the heterotic case where $\xi$ is necessarily nonzero because of the one loop contribution. In type II theory such a contribution would have to be of nonperturbative origin.

In the heterotic theory the mass of the anomalous gauge boson was proportional to the value of $\xi$. If a similar result would hold in the orientifold picture, this would mean that some of the $U(1)$ gauge bosons could become arbitrary light or even massless, a situation somewhat unexpected from our experience with consistent quantum field theories. In any case, a careful reevaluation of several questions is necessary in the light of this new situation. Among those are: the size of the $\xi$ 's, the size of the masses of anomalous $U(1)$ gauge bosons, the relation of $\xi$ and gauge boson mass, as well as the fate of heterotic - type IIB orientifold duality, which we will discuss in the remainder of this talk.

The questions concerning the anomalous gauge boson masses have been answered in [1] ically they are large, of order of the string scale, even if the corresponding Fayet-Iliopoulos terms vanish. This is in agreement with the field theoretic expectation that the masses of anomalous gauge bosons cannot be small or even zero. There is one possible exception, however. In the limit that gauge coupling constant tends to zero, one could have vanishing masses. In this case, one would deal with a global U(1) that can be tolerated in field theory even if it is anomalous.

\section{Heterotic-Type I/II Duality}

Models containing anomalous $U(1)$ factors offer an arena to study details of Type I/II - Heterotic duality in four dimensions. This duality, is of the weak coupling - strong coupling type in ten dimensions. In four dimensions the relation between the heterotic and type I dilaton is

$$
\phi_{H}=\frac{1}{2} \phi_{I}-\frac{1}{8} \log \left(G_{I}\right)
$$

where $G_{I}$ is the determinant of the metric of the compact $6 \mathrm{~d}$ space, which depends on moduli fields. For certain relations between the dilaton and these moduli fields we thus have a duality in four dimensions which maps a weakly coupled theory to another weakly coupled theory.

For the remainder of the discussion we have to be very careful with the definition of heterotic - type I duality. Such a duality has first been discussed in [23i] in ten dimensions. It was explicitely understood as a duality between the original $S O(32)$ type I theory and the heterotic theory with the same gauge group, that is a duality between two theories that both have one antisymmetric tensor field in ten dimensions. This is a very well established duality symmetry which will not be the focus of our discussion here. We would like to concentrate on a four dimensional duality symmetry between more general type II 
orientifolds and the heterotic $S O(32)$ theory first discussed in [200 orientifold duality. It would relate theories that have a different number of antisymmetric tensor fields in their ten dimensional origin.

The pairs of models which we study are type IIB orientifolds models in $4 \mathrm{~d}$ and their candidate heterotic duals which can be found in the existing

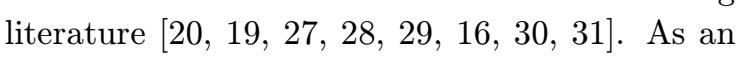
example consider the $Z_{3}$ orientifold/orbifold [ $[201]$.

On one side, the type IIB orientifold model has the gauge group $G=S U(12) \times S O(8) \times$ $U(1)_{A}$ where the $U(1)_{A}$ factor is anomalous. The anomalies are non-universal and get cancelled by means of the generalized Green-Schwarz mechanism. This mechanism involves twenty-seven twisted singlets $M_{\alpha \beta \gamma}$, a particular combination of which combines with the anomalous vector superfield to form a massive multiplet. After the decoupling of this heavy vector multiplet we obtain the nonanomalous model with the gauge group $G^{\prime}=S U(12) \times S O(8)$.

On the other side, with the heterotic $S O(32)$ superstring compactified on the orbifold $T^{6} / Z_{3}$, the gauge group is $G=S U(12) \times S O(8) \times U(1)_{A}$ and the $U(1)_{A}$ is again anomalous. Its anomalies, however, are universal in this case, and a universal, only dilaton-dependent, Fayet-Iliopoulos term is generated. In this case there are also fields which are charged only under the anomalous $U(1)$ that can compensate for the FayetIliopoulos term by assuming a nontrivial vacuum expectation value, without breaking the gauge group any further; a combination of these fields and of the dilaton supermultiplet is absorbed by the anomalous vector multiplet. These nonabelian singlets are the counterparts of the $M_{\alpha \beta \gamma}$ moduli of the orientifold model. However, on the heterotic side we have additional states $V$ charged under $U(1)_{A}$ (and also under $S O(8)$ ) the counterparts of which are not present in the orientifold model. These unwanted states become heavy in a supersymmetric manner through the superpotential couplings $[\overline{1} \overline{9} \overline{1}$. Below the scale of the heavy gauge boson mass we have a pair of models whose spectra fulfil the duality criteria.

One should note that on the heterotic side we have a blown-up orbifold, since the scalars that assume a vacuum expectation value corre- spond to the blowing-up modes. Thus, in this case, a Type IIB orientifold is found to be dual to a blown-up heterotic orbifold * . The next point to be stressed is that this duality works even though no Fayet-Iliopoulos term is present on the orientifold side. In Ref. [1 $\left.19 \overline{9}_{1}^{\prime}\right]$ where, according to the general belief, the generation of a 1-loop Fayet-Iliopoulos term in the orientifold model had been assumed, duality held only in a region of the moduli space where the nonabelian gauge groups are broken. If such a term were generated on the Type IIB side, perhaps by a nonperturbative mechanism, the duality would still hold, but one would have to blow up the orientifold on the Type IIB side.

There exist, however, examples where exact duality apparently cannot be achieved [i]n. The first of the examples that was found to show this behaviour is the $Z_{7}$ orientifold/orbifold model given in [2글. The orientifold model has the gauge group $G=S U(4)^{3} \times S O(8) \times U(1)^{3}$. All three $U(1)$ factors are anomalous and their gauge bosons decouple upon getting masses by the nonuniversal Green-Schwarz mechanism. These gauge bosons mix with combinations of the chiral superfields $M$ in the Ramond-Ramond sector which transform nonlinearly under the $U(1)$ 's. In this case the unbroken gauge group is large, $G^{\prime}=$ $S U(4)^{3} \times S O(8)$, since the inspection of the potential shows that the charged fields are not forced to assume vacuum expectation values breaking the nonabelian subgroups. The situation is very different on the heterotic orbifold side. Here we have a unique anomalous $U(1)$ and a FayetIliopoulos term $\xi^{2} \propto \operatorname{Tr} Q>0$. The only fields at hand which can cancel the anomalous D-term and participate in giving a mass to the gauge boson are not only charged under the anomalous $\mathrm{U}(1)$ but are also charged under the $S U(4)^{3}$ nonabelian factor. Thus this group is spontaneously broken together with the nonanomalous $U(1)$ at the string scale, and the low-energy gauge group is different from that on the Type IIB side, in contradiction to the conjectured duality symmetry. The second problematic aspect is that those fields $M$ of the heterotic model (that must ac-

*The blowing-up of the $Z_{3}$ orientifold has been recently discussed in Ref. [35]. 
quire vevs in order to render other states $V$ massive that are not present in the orientifold model) do not have the appropriate partners in the dual model. On the orientifold side the corresponding $M$ states are gauge singlets and nothing forces them to assume nonzero vacuum expectation values.

Thus, in the $Z_{7}$ example neither the low energy gauge groups nor the massless spectra match in the supposedly dual pair, at least at the level of the perturbative effective lagrangian we rely on here. The question is whether a nonperturbative contribution to the superpotential or, perhaps a nontrivial Kähler potential dependence on the fields $M$ would change the picture. The second type of corrections, although somewhat exotic in details, could achieve duality. This comes from the fact that certain additional contributions to the Kähler potential would enforce nonzero vevs for the $M$ states on the Type IIB side and then the two models could appear as a dual pair. The same effect would be achieved if nonzero FayetIliopoulos terms were generated, perhaps by nonperturbative effects.

Therefore the naive duality conjecture does not seem to be universally valid. The first doubts reported here came from a study of the $Z_{7}$ examples [1] $\left.{ }_{1}^{1}\right]$. The spectra of the two candidate duals do not match for certain isolated values of the moduli fields. Meanwhile these doubts were confirmed and extended to other cases in a calculation of gauge coupling constants [32in]. More recently, it was shown that certain global symmetries that were found to hold on the heterotic side did not have counterparts in the orientifold picture. For a detailed discussion see [i2].

\section{Outlook}

From the fact that this duality symmetry is not universally valid we would then expect different phenomenological properties of anomalous U(1)'s

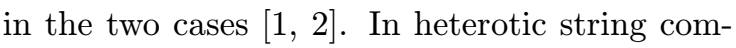
pactifications, the presence of an anomalous $U(1)$ shows up primarily in the existance of a nonvanishing Fayet-Iliopoulos term $\xi$. If such a term is somewhat smaller than the Planck scale this could explain the origin and hierarchies of the small dimensionless parameters in the low-energy lagrangian, such as the Yukawa couplings [1], in terms of the ratio $\xi / M_{P l}$. In explicit string models, $\xi$ is found to be of the order of magnitude necessary to account for the value of the Cabibbo angle. Furthermore, the universality of the mixed gauge anomalies implies a successful relation between the value of the weak mixing angle at unification and the observed fermion mass hierarchies [36]. The anomalous $U(1)$ could also play an important role in supersymmetry breaking: not only does it take part in its mediation from the hidden sector to the observable sector (as implied by the universal Green-Schwarz relation among mixed gauge anomalies), but also it can trigger the breaking of supersymmetry itself, due to an interplay between the anomalous $D$-term [1 127$]$ and gaugino condensation [1 $\left.{ }_{1}^{1} \overline{4}_{1}^{1}\right]$. It would be interesting to look at this questions in the framework of the heterotic $E_{8} \times E_{8}$ M-theory [24] in the presence of anomalous $U(1)$ symmetries, generalizing previous results of supersymmetry breakdown [25i]. Cosmologically, the presence of an anomalous $U(1)$ might have important applications in the discussion of inflationary models: in particular its Fayet-Iliopoulos term can dominate the vacuum energy of the early universe, leading to so-called D-term inflation i, Finally, the heterotic anomalous $U(1)$ might be at the origin of a solution of the strong $\mathrm{CP}$ problem [ị], while providing an acceptable dark matter candidate.

Since there is no exact heterotic - type II orientifold duality one may now ask whether the anomalous $U(1)$ 's present in type IIB orientifolds are likely to have similar consequences - or even have the potential to solve some of the problems encountered in the heterotic case. Certainly, the implications will differ somewhat. In the heterotic case, the phenomenological implications of the $U(1)_{X}$ rely on the appearance of a FayetIliopoulos term whose value, a few orders of magnitude below the Planck mass, is fixed by the anomaly. The situation is different in in the orientifold case, where the Fayet-Iliopoulos terms are moduli-dependent. The freedom that is gained by the possible adjustment of the Fayet-Iliopoulos term allows, for example, to cure the prob-

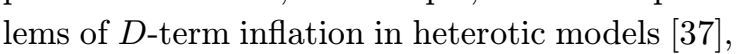
where $\xi$ turned out to be too large. 
This possible choice of $\xi$ is payed for by a loss of predictivity. In that respect, one may conclude that the orientifold anomalous $U(1)$ 's are not that different from anomaly-free $U(1)$ 's, whose Fayet-Iliopoulos terms are unconstrained and can be chosen at will. This might also influence the possible use of these $U(1)$ 's for an axion solution of the strong CP-problem. Still, these anomalous $\mathrm{U}(1)$ symmetries might play an important role in phenomenological applications.

\section{Acknowledgements}

I would like to thank Z. Lalak and S. Lavignac for interesting discussions and collaboration. This work was partially supported by funds from the European Commission programs ERBFMRX-CT96-0045 and CT96-0090.

\section{References}

[1] Z. Lalak, S. Lavignac and H.P. Nilles, hepth/9903160; Nucl. Phys. B559 (1999) 48

[2] Z. Lalak, S. Lavignac and H.P. Nilles, hepth/9912206

[3] L. Alvarez-Gaume and E. Witten, Nucl. Phys. B234 (1984) 269.

[4] W. Fischler, H.P. Nilles, J. Polchinski, S. Raby and L. Susskind, Phys. Rev. Lett. 47 (1981) 757.

[5] M. Green and J. Schwarz, Phys. Lett. B149 (1984) 117.

[6] T. Kobayashi and H. Nakano, Nucl. Phys. B496 (1997) 103.

[7] M. Dine, N. Seiberg and E. Witten, Nucl. Phys. B289 (1987) 317.

[8] J. Atick, L. Dixon and A. Sen, Nucl. Phys. B292 (1987) 109; M. Dine, I. Ichinose and N. Seiberg, Nucl. Phys. B293 (1987) 253.

[9] J.E. Kim, Phys. Lett. B207 (1988) 434; E.J. Chun, J.E. Kim and H.P. Nilles, Nucl. Phys. B370 (1992) 105; H. Georgi, J.E. Kim and H.P. Nilles, Phys. Lett. B437 (1998) 325.

[10] A. Font, L.E. Ibanez, H.P. Nilles and F. Quevedo, Nucl. Phys. B307 (1988) 109; ibid., B310 (1988) 764.

[11] L. Ibáñez and G. G. Ross, Phys. Lett. B332 (1994) 100; P. Binétruy and P. Ramond, Phys.
Lett. B350 (1995) 49; V. Jain and R. Shrock, Phys. Lett. B352 (1995) 83; E. Dudas, S. Pokorski and C.A. Savoy, Phys. Lett. B356 (1995) 45; P. Binétruy, S. Lavignac, and P. Ramond, Nucl. Phys. B477 (1996) 353;

[12] P. Binétruy and E. Dudas, Phys. Lett. B389 (1996) 503; G. Dvali and A. Pomarol, Phys. Rev. Lett. 77 (1996) 3728; Z. Lalak, Nucl. Phys. B521 (1998) 37; N. Arkani-Hamed, M. Dine, S. P. Martin, Phys. Lett. B431 (1998) 329; T. Barreiro, B. de Carlos, J.A. Casas, J.M. Moreno, Phys. Lett. B445 (1998) 82.

[13] J.A. Casas and C. Muñoz, Phys. Lett. B216 (1989) 37; J.A. Casas, J.M. Moreno, C. Muñoz and M. Quiros, Nucl. Phys. B328 (1989) 272; P. Binétruy and G. Dvali, Phys. Lett. B388 (1996) 241; E. Halyo, Phys. Lett. B387 (1996) 43.

[14] H. P. Nilles, Phys. Lett. B115 (1982) 193; Int. J. Mod. Phys. A5 (1990) 4199

[15] A. Sagnotti, in Cargese '87, "Non-Perturbative Quantum Field Theory", eds. G. Mack et al. (Pergamon Press, Oxford, 1988), p. 251; P. Horava, Nucl. Phys. B327 (1989) 461, Phys. Lett. B231 (1989) 251.

[16] G. Aldazabal, A. Font, L.E. Ibanez, and G. Violero, Nucl. Phys. B536 (1998) 29.

[17] A. Sagnotti, Phys. Lett. B294 (1992) 196.

[18] M. Berkooz, R.G. Leigh, J. Polchinski, J.H. Schwarz, N. Seiberg and E. Witten, Nucl. Phys. B475 (1996) 115.

[19] Z. Kakushadze, Nucl. Phys. B512 (1998) 221.

[20] C. Angelantonj, M. Bianchi, G. Pradisi, A. Sagnotti, and Ya.S. Stanev, Phys. Lett. B385 (1996) 96.

[21] L.E. Ibanez, R. Rabadan and A.M. Uranga, Nucl. Phys. B542 (1999) 112

[22] E. Poppitz, Nucl. Phys. B542 (1999) 31

[23] J. Polchinski and E. Witten, Nucl. Phys. B460 (1996) 525

[24] P. Horava and E. Witten, Nucl. Phys. B460 (1996) 506; Nucl. Phys. B475 (1996) 94

[25] H. P. Nilles, M. Olechowski and M. Yamaguchi, Phys. Lett. B415 (1997) 24; Nucl. Phys. B484 (1997) 33; Nucl. Phys. B530 (1998) 43;

[26] L.E. Ibanez, C. Munoz and S. Rigolin, Nucl. Phys. B553 (1999) 43

[27] Z. Kakushadze and G. Shiu, Phys. Rev. D56 (1997) 3686. 
[28] Z. Kakushadze and G. Shiu, Nucl. Phys. B520 (1998) 75.

[29] L.E. Ibanez, JHEP 9807 (1998) 002.

[30] Z. Kakushadze, G. Shiu and S.-H.H. Tye, Nucl. Phys. B533 (1998) 25.

[31] J. Lykken, E. Poppitz and S.P. Trivedi, Nucl. Phys. B543 (1999) 105

[32] I. Antoniadis, C. Bachas and E. Dudas, Nucl. Phys. B560 (1999) 93

[33] L. E. Ibanez, R. Rabadan and A.M. Uranga, hep-th/9905098

[34] C. A. Scrucca and M. Serone, hep-th/9912108

[35] M. Cvetic, L. Everett, P. Langacker and J. Wang, JHEP 9904:020,1999

[36] L. Ibáñez, Phys. Lett. B303 (1993) 55; P. Binétruy and P. Ramond, Phys. Lett. B350 (1995) 49.

[37] E. Halyo, Phys. Lett. B454 (1999) 223 